\title{
Pareto Optimal Reconfiguration of Power Distribution Systems Using a Genetic Algorithm Based on NSGA-II
}

\author{
Bogdan Tomoiagă ${ }^{1}{ }^{*}$, Mircea Chindriş ${ }^{1}$, Andreas Sumper ${ }^{2,3}$, Antoni Sudria-Andreu ${ }^{2}$ and \\ Roberto Villafafila-Robles ${ }^{2}$
}

1 Power Systems \& Management Department, Technical University of Cluj-Napoca, Memorandumului st., No. 28, 400114 Cluj-Napoca, Romania;

E-Mail: mircea.chindris@enm.utcluj.ro

2 Centre of Technological Innovation in Static Converters and Drives, Department of Electrical Engineering, College of Industrial Engineering of Barcelona, Universitat Politècnica de Catalunya-BarcelonaTech, Carrer Comte d'Urgell, 187-08036 Barcelona, Spain;

E-Mails: andreas.sumper@upc.edu (A.S.); antoni.sudria@upc.edu (A.S.-A.); roberto.villafafila@upc.edu (R.V.-R.)

3 IREC Catalonia Institute for Energy Research, Jardins de les Dones de Negre 1, 08930 Sant Adrià de Besòs, Barcelona, Spain

* Author to whom correspondence should be addressed; E-Mail: bogdan.tomoiaga @enm.utcluj.ro; Tel.: +40-264401451; Fax: +40-364819656.

Received: 17 December 2012; in revised form: 2 February 2013 / Accepted: 19 February 2013 / Published: 6 March 2013

\begin{abstract}
Reconfiguration, by exchanging the functional links between the elements of the system, represents one of the most important measures which can improve the operational performance of a distribution system. The authors propose an original method, aiming at achieving such optimization through the reconfiguration of distribution systems taking into account various criteria in a flexible and robust approach. The novelty of the method consists in: the criteria for optimization are evaluated on active power distribution systems (containing distributed generators connected directly to the main distribution system and microgrids operated in grid-connected mode); the original formulation (Pareto optimality) of the optimization problem and an original genetic algorithm (based on NSGA-II) to solve the problem in a non-prohibitive execution time. The comparative tests performed on test systems have demonstrated the accuracy and promptness of the proposed algorithm.
\end{abstract}


Keywords: power distribution systems; reconfiguration; genetic algorithms; multi-objective optimization; pareto optimality

\section{Introduction}

The most important measures which can improve the performance in the operation of a distribution system are: (i) reconfiguration of the system, exchanging the functional links between its elements (system/network/feeder reconfiguration problem); (ii) variation and control of the reactive power flow through the system (optimal reactive power dispatch problem), using bank capacitors, power generators, etc.; (iii) variation and control of the voltage by using on-load tap-changers for power transformers (by using automatic voltage regulators); and (iv) changing the operating scheme of the parallel connected power transformers, etc. This paper focuses on optimization through the reconfiguration of power distribution systems.

The reconfiguration problem is one of the multi-criteria optimization types, where the solution is chosen after the evaluation of some indices (e.g., active power losses, reliability indices, branch load limits, voltage drop limits, etc.), which represent multiple purposes. These criteria can be grouped in two different categories: (i) objective functions: criteria that must be minimized; and (ii) constraints (restrictions): criteria that must be included within some bounds. On the other hand, the criteria are incompatible from the point of view of measurement units and are often conflicting. Moreover, some criteria can be (or it is important for them to be) modeled, at the same time as objectives and constraints. For instance, the active power losses must be minimized but we can simultaneously impose a maximal acceptable value (constraint). Thus, in order to solve the problem, first of all, $a$ proper model has to be chosen. The problem of optimization through the reconfiguration of a power distribution system, in terms of its definition, is a historical single objective problem with constraints. Since 1975, when Merlin and Back [1] introduced the idea of distribution system reconfiguration for active power loss reduction, until nowadays, a lot of researchers have proposed diverse methods and algorithms to solve the reconfiguration problem as a single objective problem. The most frequently used one is the main criterion method ( $\varepsilon$-constraint) where the problem is defined in the following conditions: a main criterion is chosen, concomitantly indicating acceptable values for the other criteria. Usually, active power losses are adopted as the main criterion [1-22]. This approach has a major weakness because there is more than one index that must be taken into account in the optimization process and, without any prior information about the different criteria, choosing the acceptable value can be problematic. Additionally, this approach alters the essence of the original technical problem. On the other hand, some authors have studied this problem using aggregation functions, converting the multi-objective problem into a single objective one that assumes a (weighted or not) sum of the selected objective functions [23-30]. The major difficulty in this kind of problem consists in the incompatibility of different criteria. To create a global function, all criteria must be converted to the same measurement unit; a frequently used method is to convert them into costs, which is usually a tricky and often inaccurate operation. In addition, subjectivity appears, caused by the introduction of weighting factors for different criteria. Thus, the existence of a model that could take into 
consideration more objective functions and constraints at the same time is of great interest. To eliminate the subjectivity and rigidity of the classic methods, the authors propose an original approach to formulate this problem using the Pareto optimality concept that defines a dominate relation among solutions.

Regardless of the problem formulation, searching for the solution is also a very complex issue due to its combinatorial nature. An absolute (e.g., "brute force" [10]) method which generates the entire space of candidate solutions in order to choose the best one, requires a prohibitive execution time. In order to avoid the evaluation of the entire space of the candidate solutions and to minimize the computation burden, several algorithms have been developed. Most authors have used different well known heuristics (branch exchange [2,3,21], branch and bound [1,4], simulated annealing [5]), other heuristic rules or meta-heuristics [7-9,11-13,15,17,22,23,25,27,28] or multi-agent technologies [20]. On the other hand, some authors have developed methods based on evolutionary computation techniques [6,14,16,18,19,24,26,29,30]. An important drawback of these methods is the fact that they solve the reconfiguration problems as single objective problems. Nevertheless, some authors have proposed Pareto optimality based approaches (including active power losses and reliability indices as objectives). With these approaches, linear programming cannot be used because we have more than one objective function. Thereby, different artificial intelligence based methods have been used: evolutionary [31], branch exchange [32] and particle swarm optimization [33].

Taking into account these considerations, we can observe the fact that this problem is arduous particularly from two points of view: (i) the formulation of the problem, because there is more than one objective; (ii) the search for the optimal solution, because of the prohibitive execution time demanded by applying an absolute method. In this paper an original method, aiming the optimization through the reconfiguration of distribution systems, is proposed. The novelty of the method consists in:

- the criteria for optimization are evaluated on active power distribution systems (containing distributed generators connected directly to the main distribution system and microgrids operated in grid-connected mode);

- the original formulation of the optimization problem, as a Pareto optimal one, with two objective functions (active power losses and system average interruption frequency index);

- an original genetic algorithm (based on NSGA-II) to solve the problem (as a Pareto optimal one) in a non-prohibitive execution time.

\section{Problem Formulation}

\subsection{Criteria for Optimization}

\subsubsection{Active Power Losses $(\Delta P)$}

Active power losses represent the most important criterion and cannot be ignored in reconfiguration problems [1-33]. In order to evaluate this criterion it is necessary to perform the load flow calculus. Basically, power flow algorithms are iterative and are based on different procedures: Gauss-Seidel, Newton-Raphson, backward/forward sweep. For distribution systems which are operated in radial configurations, the most recommended approaches are backward/forward sweep based algorithms. 
Due to its accuracy, the relatively small iteration number required and its robust convergence, the version presented in [34] has been chosen (where the sources are modeled as PQ nodes).

\subsubsection{Reliability of the Distribution System}

The essential attributes of interruptions in the power supply of the customers are the frequency and duration. While duration is predominantly influenced by the distribution system structure (radial, meshed, weak meshed) and the existing automations, the frequency is mainly influenced by the adopted operational configuration; it can be minimized by the suitable choice of the effective configuration. In other words, through reconfiguration, we can improve those reliability indices which refer to the interruption frequency [32]. Otherwise, the reliability of a distribution system can be considered from two different angles:

- Reliability of a particular customer: e.g., the average number of interruptions to the power supply. This index can represent a possible objective and/or constraint in the optimization problem (because some customers can impose maximal/minimal limits in their supply contracts);

- Reliability of the entire supply system: e.g., the number of interrupted customers per year [32], system average interruption frequency index (SAIFI) [35] (defined as: total number of customer interruptions longer than 3 minutes per total number of customers served).

For any fault that will lead to the interruption of the power supply from the main distribution system, the existing distributed generators will be switched off owing to a variety of reasons. We point out only two of them: (i) an operation of a power island purely with dispersed generators is usually considered unacceptable; (ii) it is important to create conditions for auto-reclose of the main circuit breaker (if this equipment is used in the distribution system). An exception appears if we have microgrids connected to the distribution network (which contains controllable loads, DG sources, storage devices, automations, etc.). In this case the consumers (loads) from the microgrid will be supplied even if a fault has occurred in the distribution system. For instance, if a fault occurred on the electric line 4-7 (Figure 1), the DG connected to node 4 and the microgrid connected to node 3 will be isolated (e.g., by loss of mains protection [36]). In this case, the microgrid is expected to operate as a power island (even if is necessary to operate the automatic load-shedding control equipment). The customers from the microgrid will be supplied even if a fault has occurred in the main distribution system because a microgrid must have both primary and secondary control algorithms [37].

Figure 1. A distribution smart grid with a distributed generator and a microgrid.

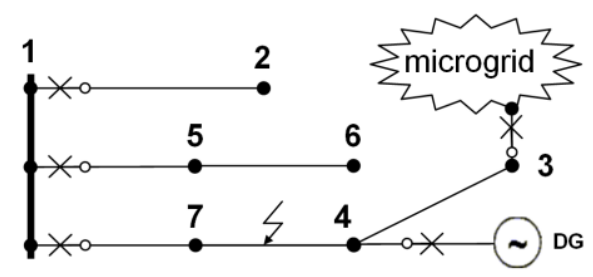

Knowing the failure rates at the level of each supplied node (load point), we can estimate the SAIFI using the relationship (in a similar form to the one given in [38]): 


$$
S A I F I=\frac{\sum_{i=1}^{n} \lambda_{t i} \cdot N_{i}}{N} \cdot T
$$

where $N$ represents the total number of customers served; $N_{i}$ is the total number of customers supplied from node $i$; $T$ is the reference period [year], ( $T=1$ year $) ; n$ is the number of load nodes of the system; $\lambda_{\mathrm{ti}}$ is the total failure rate of the equivalent element corresponding to the reliability block diagram at the level of node $i\left[\right.$ year $\left.^{-1}\right]$.

The total failure rate at the level of a node $i$ (for a configuration) can be calculated as $\lambda_{\mathrm{ti}}=\lambda_{\mathrm{i}}+k \lambda_{\mathrm{i}}$, where $\lambda_{\mathrm{i}}$ is the failure rate when the restoring of supply is performed after the fault repair; while $\lambda_{\mathrm{i}}{ }^{\prime}$ is the failure rate when the restoring of supply is performed after the fault isolation through non-automatic maneuvers; and $k=[0,1]$ is a coefficient that estimates the weight of restoring after 3 minutes (sustained interruption restored after fault isolation). In a first approximation, for reconfiguration studies, we consider $k=1$, which corresponds to the most unfavorable case (when all restorations of supply after fault isolations through non-automatic maneuvers are performed after 3 minutes) [31]. It must be also specified that the contingencies are considered simple and the electric distribution system components are independent from the reliability point of view. A numerical example in order to explain how the SAIFI index is computed is given in what follows. Considering the sample system from Figure 1 we assumed that each branch has the failure rate $\lambda=0.5$ [year ${ }^{-1}$, on each supplied node there are 30 customers and the microgrid connected to node 3 (which contains 30 customers and DG units) behaves as a source. In this case we will have ( $T=1$ year):

- $\quad N=30 \cdot 6=180$ (six nodes, 30 customers for each node);

- $\lambda_{\mathrm{t} 2} \cdot N_{2}=0.5 \cdot 30=15$

- $\lambda_{\mathrm{t} 3} \cdot N_{3}=0.5 \cdot 3 \cdot 0=0$ (three branches, the microgrid is expected to operate as an island and customers from a microgrid will be supplied even if a fault has occurred in the main distribution system);

- $\quad \lambda_{\mathrm{t} 4} \cdot N_{4}=0.5 \cdot 3 \cdot 30=45$ (three branches, if a fault occurs on the path 1-7-4-3, the DG unit will be switched off and the customers will be not supplied);

- $\lambda_{\mathrm{t} 5} \cdot N_{5}=\lambda_{\mathrm{t} 6} \cdot N_{6}=0.5 \cdot 2 \cdot 30=30$ (two branches);

- $\lambda_{\mathrm{t} 7} \cdot N_{7}=0.5 \cdot 3 \cdot 30=45$;

- $S A I F I=(15+0+45+30+30+45) / 180=0.9167$.

\subsubsection{Other Criteria}

- Node Voltages $\left(V_{i}\right)$ : Basically, each voltage r.m.s. value of the network nodes must be framed within the allowable limits.

- Branch Load Limits through Lines $\left(I_{i j}\right)$ : a typical constraint on the reconfiguration problem.

- Safeguard of power supplies for all customers: The attached graph of the electric system should be connected (a tree or a forest).

- Configuration of the Distribution System: Generally, electrical distribution systems are operated in radial configuration. This condition can be expressed as follows: 


$$
\sum_{i j \in E} \alpha_{i j}=n-p
$$

where $\alpha_{i j}$ is a binary variable, representing the status of a tie line ( $0-$ open, $1-$ closed); $n$ is the number of electric system nodes; $E$ is the set of power system lines (branches) and $p$ is the number of connected components. In graph theory terms, for a system with one source $(p=1)$ we are talking about an optimal tree and for a system with more than one feeder $(p>1)$ we are talking about an optimal forest with a number of trees (connected components) equal to that of source nodes.

\subsection{Pareto Optimality Problem Formulation}

The criteria presented above are not unique, but we consider them to be the most important ones. Taking into account these criteria, we can begin to perceive the real dimensions of the problem. These criteria are incompatible from the point of view of measurement units and can be grouped in two different categories: objective functions and constraints (restrictions). In Pareto optimization, the central concept is named non-dominated solution. This solution must satisfy the following two conditions: (i) there is no other solution that is superior at least in one objective function; (ii) it is equal or superior with respect to other objective function values. Usually, the solution is not unique and consists of a set of acceptable optimal solutions (Pareto optimal). The set of Pareto solutions forms the Pareto front associated with a problem. Figure 2 presents a possible Pareto front for the optimization problem based on two objectives ( $\triangle P$ and $S A I F I)$. The Pareto front allows an informed decision to be made by visualizing an extensive range of options since it contains the solutions that are optimal from an overall standpoint.

Figure 2. A Pareto front for a bi-objective reconfiguration problem.

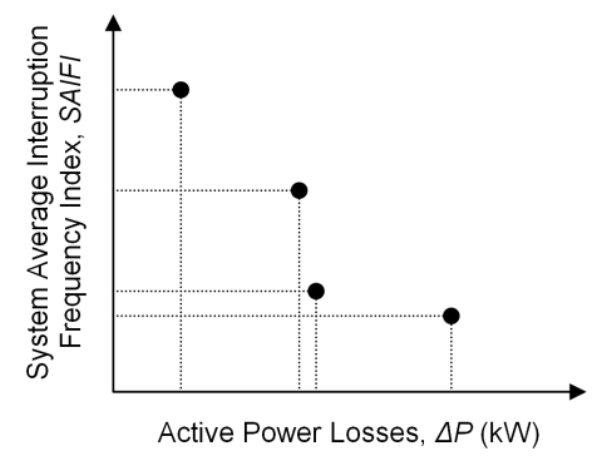

As a Pareto optimal multi-objective problem, we propose the following form:

Objective function

$$
\min [\triangle P, S A I F I]
$$

Constraints

$$
\begin{aligned}
& V_{i}^{\min } \leq V_{i} \leq V_{i}^{\max } \\
& I_{i j} \leq I_{i j}^{\max } \\
& \sum_{i j \in E} \alpha_{i j}=n-p
\end{aligned}
$$




\section{Problem Solving}

In the case of multi-objective problems, the literature proposes several Pareto-based genetic algorithms: MOGA (Multi Objective Genetic Algorithm) [39], NPGA (Niched Pareto Genetic Algorithm) [40], SPEA/SPEA-II (Strength Pareto Evolutionary Algorithm) [41], and NSGA/NSGA-II (Non-dominated Sorting Genetic Algorithm) [42]. After experimental comparison of these algorithms, the authors have decided to solve the multi-objective optimization problem through reconfiguration by using the NSGA-II algorithm with significant results (optimal solutions in short execution times). The logical diagram of the proposed NSGA-II based algorithm (MOReco-Multi Objective Reconfiguration) is given in Figure 3. The initial population is generated using the branch-exchange heuristic algorithm presented in [3]. A potential solution is selected only if all considered constraints are satisfied.

Figure 3. Logical diagram of the proposed algorithm (MOReco) dedicated to the reconfiguration of power distribution systems.

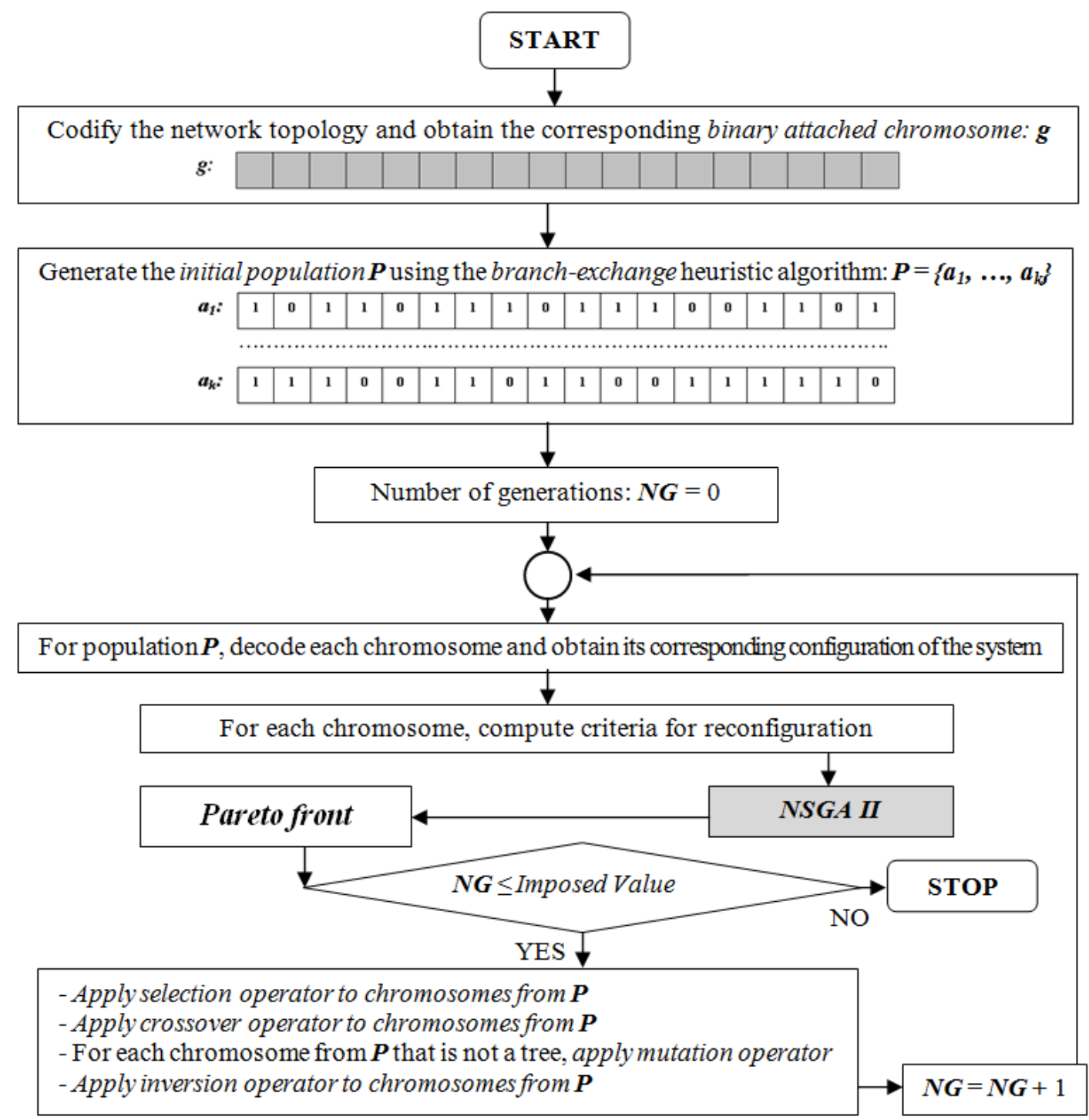




\subsection{Genetic Encoding and Decoding of Power Distribution System Topology}

\subsubsection{Genetic Encoding}

In this implementation, the representation using the branch lists was chosen because a power system node is only linked with a small part of the other nodes (it results in a rare graph, i.e., the associated matrix contains many zero elements). Consequently, the graph associated with the electric network can be described by a matrix with 2 lines and $m$ columns (where $\mathrm{m}$ is the number of the branches), each column indicating the two ends of a branch. This matrix does not contain zero elements. Therefore, using the representation via the branch lists, a binary codification of the problem (binary chromosome with fixed length) can be obtained. Binary values of the chromosome will indicate the status of every electric line: 0-open, 1-closed. Figure 4a exemplifies the graph (which indicates the network topology) attached to a distribution system, represented by branch lists ( $\alpha$ and $\beta$ ), and the binary attached chromosome $\mathrm{g}$ (system/grid encoding).

Figure 4. A power distribution system: (a) the branch lists of the attached graph ( $\alpha$ and $\beta$ ) and the attached chromosome $(g)$; (b) Branch lists $(\alpha$ and $\beta$ ) obtained by decoding the chromosome $a$.

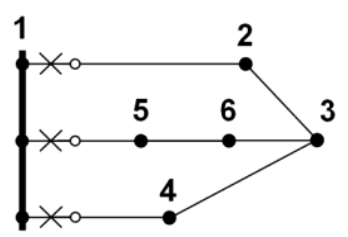

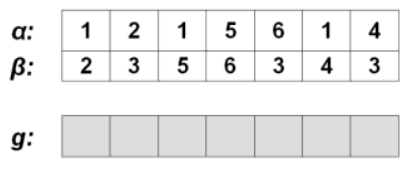

(a)
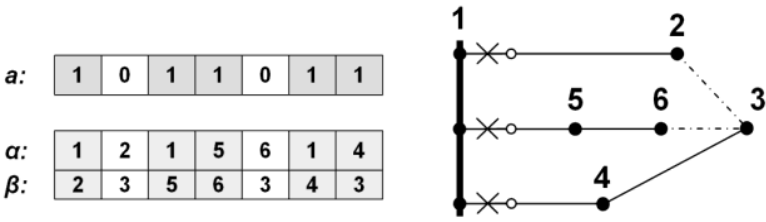

(b)

\subsubsection{Genetic Decoding}

The operation scheme of the system will be obtained by making the preservation of the corresponding branch value equal 1 (in operation). For instance, by decoding the chromosome a, the radial operation scheme will be obtained (with corresponding $\alpha$ and $\beta$ lists) (Figure $4 \mathrm{~b}$ ). Using this codification, we have a population that consists of a set of chromosomes of type a. By decoding each chromosome, a particular configuration will be obtained and its performance can be tested.

\subsection{Genetic Operators}

\subsubsection{Selection}

The goal of the selection operator is to assure more chances to replicate for the best chromosomes of a population. The selection is performed taking into account the fitness of the chromosomes. The most used selection methods are Monte Carlo and tournament. For this multi-objective optimization problem, the author has used the ecological niche method [43]. 


\subsubsection{Crossover}

Choosing the number and position of crossover points for the crossover operator depends on the system topology. If these points are selected in an inadequate mode we will obtain "bad" chromosomes: (i) un-connected systems with isolated nodes; or (ii) connected systems with loops (meshed). In order to reduce the number of these cases, we propose that the number of cut points be equal to $C N-1$. CN represents the cyclomatic number (the number of fundamental circuits/loops) corresponding to the attached graph: $C N=m-n+p$ (where $m$ is the number of branches, $n$ is the number of nodes/vertices and $p$ is the number of connected components).

\subsubsection{Mutation}

One of the two conditions in order to have a tree or a forest is to assure $n$ - $p$ closed branches (in operation), as in relationship (2). A radial configuration cannot be converted to another radial one by simply altering the value of a chosen gene. Therefore, we use this operator only in the case when, by performing the crossover operator, non-radial configurations are obtained. Thus, if in a chromosome there are more or fewer than $n-p$ genes equal to 1 , the mutation operator randomly replaces the excess/insufficiency of genes equal to 1 (in order to have $n-p$ genes equal to 1 ).

\subsubsection{Inversion}

The second condition in order to have a tree or a forest is to have a connected graph (for a tree) or a graph with connected components (for a forest). Thus, this operator makes some branch-exchanges (each inversion between two genes of a chromosome behaves as a branch-exchange), repairing existing non connected graph chromosomes (which are not connected but which have $n-p$ genes equal to 1) and increases the diversity of a population. In our algorithm, this is an intensively used operator after performing crossover and mutation.

\section{Simulation Results}

The Pareto front allows an informed decision to be made by visualizing an extensive range of options since it contains the solutions that are optimal from an overall standpoint. The proposed algorithm was implemented in the $C++$ programming language. The implementation was adapted in order to work with one objective $(\triangle P)$ and with two objectives ( $\triangle P$ and SAIFI). Thus, the user can choose (in a flexible mode) $\Delta P$ as the objective function and some criteria as constraints (voltage deviation, loads limits through lines, etc.) or a vector objective function with $\triangle P$ and SAIFI as variables (at the same time) and other criteria as constraints. The stopping criterion of the algorithm is an imposed maximum number of generations. All tests were performed on a PC equipped with an Intel Core Duo $(3 \mathrm{GHz}$ ) processor and $4 \mathrm{~GB}$ of memory. Validation of the proposed algorithm implementation has been a difficult process especially because of the load flow computation unit. Data regarding different quantities (active power losses, voltages nodes) have been presented in literature in a variety of forms (p.u., actual values or a mixture) and there are some approximations caused by the load flow algorithm which was used. E.g., in case [2], before the reconfiguration (in the base case), the total active power losses are 0.00511 p.u. After reconfiguration (by applying the MOReco algorithm), 
the total active power losses are 0.004658 p.u. This result represents the optimal configuration and is confirmed in [9] and [11]. Another example is given in [5]. Before the reconfiguration, the total active power losses are $20.87 \mathrm{~kW}$. This value is different from the $69.76 \mathrm{~kW}$ value which was presented in [5]. After reconfiguration, the total active power losses are $9.43 \mathrm{~kW}$. These results are confirmed in [16].

In order to test the correctness and convergence speed of the proposed algorithm, the authors studied, first of all, five well known single-objective (active power losses) test systems. Table 1 presents two single-objective (active power losses) test cases where power distribution systems contain distributed generation:

- System A (Figure 5a) [3,27]: There are four DG units installed on nodes 3, 6, 24 and 29 [27]. In the base case, the total active power losses are $169.881 \mathrm{~kW}$. By applying MOReco algorithm (after reconfiguration), the total active power losses are $115.748 \mathrm{~kW}$. The evolution of the active power losses along the searching process is presented in Figure $6 \mathrm{a}$.

- System B (Figure 5b) [27]: In this case, there are eight DG units installed on nodes: 7, 12, 19, 28, $34,71,75$ and 79 [26]. Before the reconfiguration, the total active power losses are $425.131 \mathrm{~kW}$ (as in [26]). After reconfiguration, we obtained a better configuration than the one presented in [26]. The total active power losses are $380.656 \mathrm{~kW}$, therefore smaller than $383.524 \mathrm{~kW}$. The evolution of the active power losses along the searching process is presented in Figure 6b.

Figure 5. Test distribution systems: (a) system A [3]; (b) system B [27].

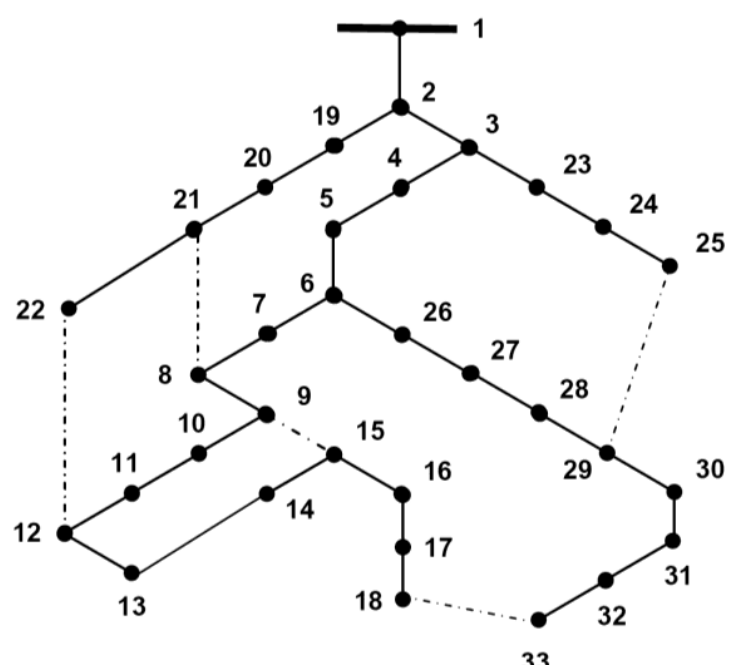

(a)

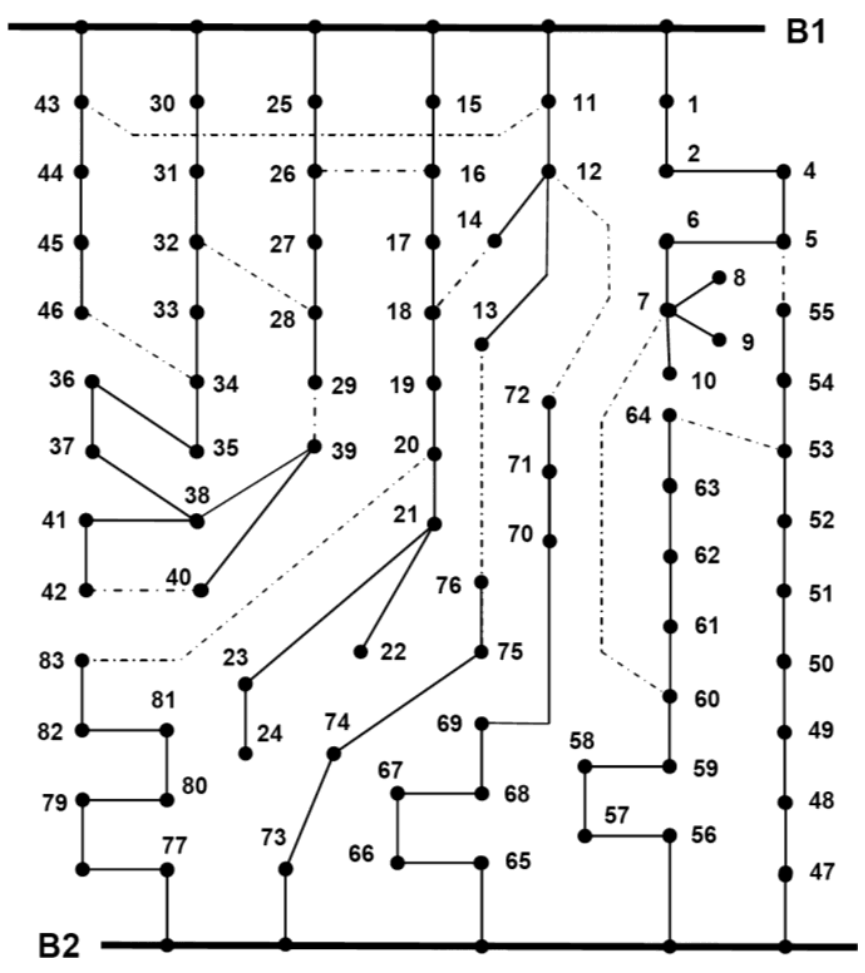

(b)

The proposed algorithm has an excellent behavior obtaining good quality solutions in reduced computation times (seconds); usually, a reduced number of generations is necessary for convergence. Performing reconfiguration for the test system B [27] as a Pareto problem, the operating configurations are obtained by optimizing two criteria: $\Delta P$ and SAIFI (Table 2). The Appendix indicates the failure 
rates of branches considered in reconfiguration calculus. It is assumed that each node has three supplied consumers. In these study cases we considered two scenarios:

Figure 6. The evolution of the active power losses along the searching process (in the case of minimum CPU runtime): (a) for test distribution system A with DGs; (b) for test distribution system B with DGs.

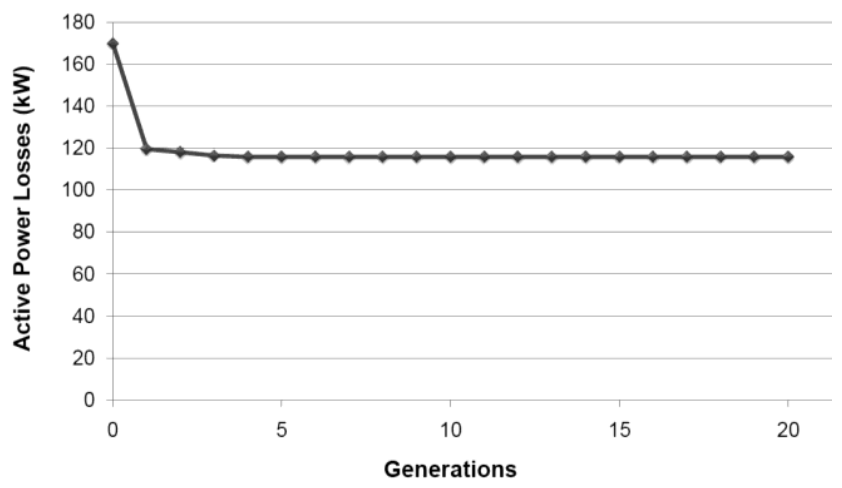

(a)

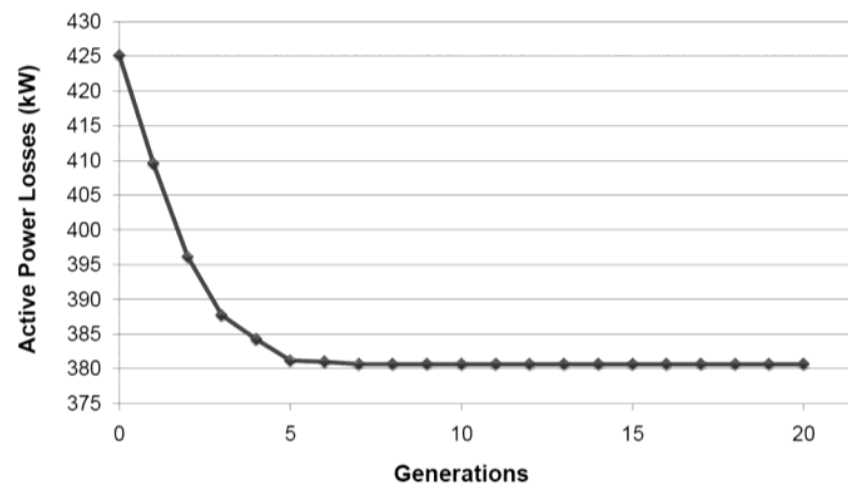

(b)

Table 1. Results for different single-objective reconfiguration (test cases with DGs).

\begin{tabular}{|c|c|c|c|c|c|c|}
\hline System & Configuration & Open branches (tie lines) & $\begin{array}{r}\text { Active p } \\
\text { losse }\end{array}$ & $\begin{array}{l}\text { ower } \\
\text { s }\end{array}$ & $\begin{array}{c}\text { CPU runtime for } \\
100 \text { runs }\end{array}$ & $\begin{array}{l}\text { Population/ } \\
\text { Generations }\end{array}$ \\
\hline \multirow[b]{2}{*}{ A } & Base case & $8-21,9-15,12-22,18-33,25-29$ & 169.881 & $\mathrm{~kW}$ & - & \\
\hline & MOReco & $7-8,9-10,14-15,28-29,32-33$ & 115.748 & $\mathrm{~kW}$ & $\begin{array}{l}\text { Minimum (3 s: } 804 \mathrm{~ms}) \\
\text { Maximum (3 s: } 974 \mathrm{~ms}) \\
\text { Average (3 s: } 910 \mathrm{~ms})\end{array}$ & $10 / 4$ \\
\hline \multirow{2}{*}{ B } & Base case & $\begin{array}{l}5-55,7-60,11-43,12-72,13-76, \\
14-18,16-26,20-83,28-32, \\
29-39,34-46,40-42,53-64\end{array}$ & 425.131 & $\mathrm{~kW}$ & - & \\
\hline & MOReco & $\begin{array}{l}6-7,12-13,32-33,38-39,41-42, \\
54-55,62-63,71-72,82-83, \\
11-43,14-18,16-26,28-32\end{array}$ & 380.656 & $\mathrm{~kW}$ & $\begin{array}{l}\text { Minimum (6 s: } 202 \mathrm{~ms}) \\
\text { Maximum (7 s: } 108 \mathrm{~ms}) \\
\text { Average (6 s: } 675 \mathrm{~ms})\end{array}$ & $10 / 8$ \\
\hline
\end{tabular}

Table 2. Results for Pareto reconfiguration with two objectives.

\begin{tabular}{|c|c|c|c|c|c|c|}
\hline System & Open branches (tie lines) & $\begin{array}{r}\text { Active p } \\
\text { losse }\end{array}$ & wer & SAIFI & $\begin{array}{l}\text { CPU runtime } \\
\text { for } 100 \text { runs }\end{array}$ & $\begin{array}{l}\text { Population/ } \\
\text { Generations }\end{array}$ \\
\hline \multirow{4}{*}{ 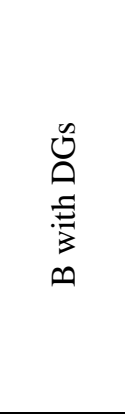 } & $\begin{array}{l}6-7,12-13,32-33,38-39,41-42,54-55,62-63, \\
71-72,82-83,11-43,14-18,16-26,28-32\end{array}$ & 380.656 & $\mathrm{~kW}$ & 1.143 & \multirow{4}{*}{$\begin{array}{c}\text { Minimum } \\
\text { (6 s: } 952 \mathrm{~ms}) \\
\text { Maximum } \\
\text { (7 s: } 279 \mathrm{~ms}) \\
\text { Average } \\
\text { (7 s: } 40 \mathrm{~ms})\end{array}$} & $10 / 9$ \\
\hline & $\begin{array}{l}5-55,6-7,11-43,12-72,13-76,14-18,16-26, \\
20-83,28-32,29-39,32-33,40-42,53-64\end{array}$ & 396.143 & $\mathrm{~kW}$ & 0.751 & & - \\
\hline & $\begin{array}{l}5-55,6-7,11-43,12-72,13-76,14-18,16-26, \\
20-83,28-32,29-39,34-46,40-42,53-64\end{array}$ & 409.526 & $\mathrm{~kW}$ & 0.648 & & - \\
\hline & $\begin{array}{l}5-55,7-60,11-43,12-72,13-76,14-18,16-26, \\
20-83,28-32,29-39,34-46,40-42,53-64\end{array}$ & 425.131 & $\mathrm{~kW}$ & 0.472 & & - \\
\hline
\end{tabular}


Table 2. Cont.

\begin{tabular}{|c|c|c|c|c|c|}
\hline System & Open branches (tie lines) & $\begin{array}{c}\text { Active power } \\
\text { losses }\end{array}$ & SAIFI & $\begin{array}{l}\text { CPU runtime } \\
\text { for } 100 \text { runs }\end{array}$ & $\begin{array}{l}\text { Population/ } \\
\text { Generations }\end{array}$ \\
\hline \multirow{3}{*}{ 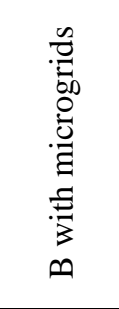 } & $\begin{array}{l}6-7,12-13,32-33,38-39,41-42,54-55,62-63, \\
71-72,82-83,11-43,14-18,16-26,28-32\end{array}$ & 380.656 & 0.988 & $\begin{array}{l}\text { Minimum } \\
\text { (7 s: } 114 \mathrm{~ms})\end{array}$ & $10 / 9$ \\
\hline & $\begin{array}{l}5-55,6-7,11-43,12-72,13-76,14-18,16-26, \\
20-83,28-32,29-39,32-33,40-42,53-64\end{array}$ & 396.143 & 0.619 & $\begin{array}{l}\text { Maximum } \\
\text { (7 s: } 342 \mathrm{~ms})\end{array}$ & - \\
\hline & $\begin{array}{l}5-55,7-60,11-43,12-72,13-76,14-18,16-26, \\
20-83,28-32,29-39,34-46,40-42,53-64\end{array}$ & 425.131 & 0.427 & $\begin{array}{c}\text { Average } \\
(7 \mathrm{~s}: 171 \mathrm{~ms})\end{array}$ & - \\
\hline
\end{tabular}

- System B with DG units: The proposed algorithm has obtained a Pareto front with four solutions (Figure 7a). In this case, the first non-dominated solution was obtained from initial population. After the first generation, the algorithm found the second non-dominated solution (the Pareto front contains two solutions). The searching process continued and the third non-dominated solution was found in generation 2 (at the end of generation 2, the Pareto front contains three solutions). The searching process continued, but without finding other non-dominated solutions until generation 9 , where the fourth and final non-dominated solution was found. In the end, the Pareto front contains four non-dominated solutions.

- System B with microgrids (instead of DG units, as sources on the points of common coupling): The proposed algorithm obtained a Pareto front with three solutions (Figure 7b). As in the previous case, along the searching process, the Pareto front increases from one solution (from the initial population), to two solutions (generation 2) and, finally, to three solutions (in generation 9). We can observe that the SAIFI index is smaller in the case of existing microgrids than in the case where we consider just distributed generators.

Figure 7. The Pareto front (in the case of minimum CPU runtime): (a) for system B with DG units; (b) for system B with microgrids.

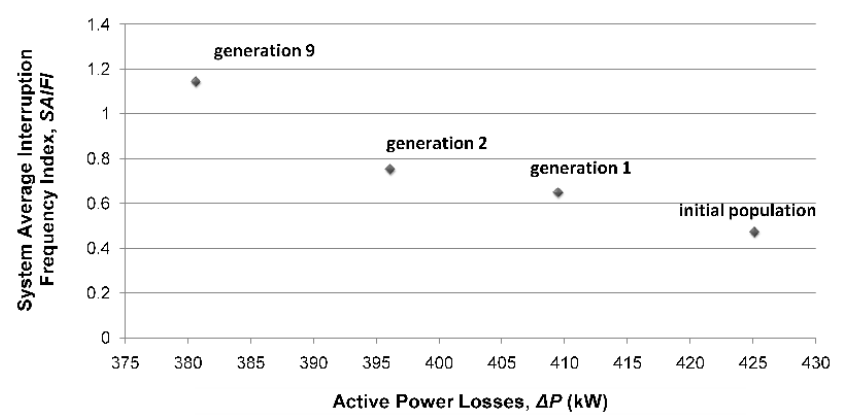

(a)

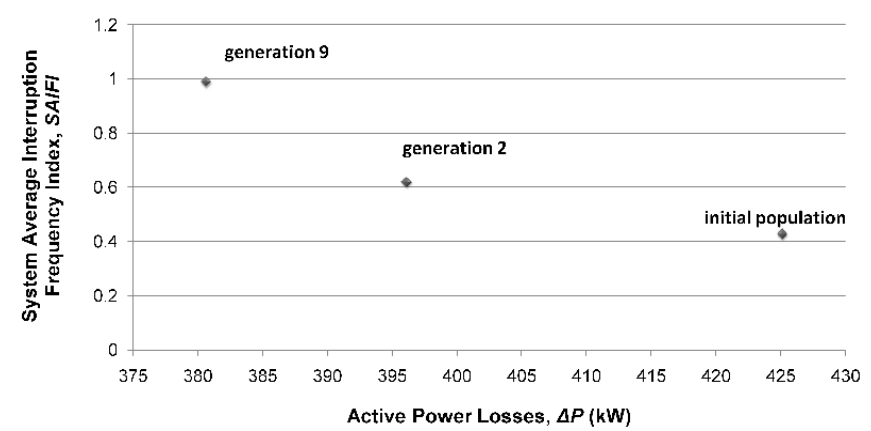

(b)

It is important to mention the fact that, in the case of the proposed algorithm, we obtained the same results for 100 runs. The differences consist only in the execution time required (e.g., between 6 s: $952 \mathrm{~ms}$ and $7 \mathrm{~s}$ : $279 \mathrm{~ms}$, for the test case B with DGs, Table 2, where multi-objective reconfiguration is done), especially because of the inversion operator (applied a random number of times). There are different execution times in a generation and an important advantage: a large breadth search and a convergence to optimum in a reduced number of generations. 
The proposed algorithm tries to exploit the fundamental properties of a distribution system, i.e., to have a radial configuration in operation. It tries to generate just radial configurations (by using the branch exchange heuristic procedure to generate the initial population and by crossover operator.) By choosing the number of cut points equal to the cyclomatic number -1 , usually, other valid chromosomes are obtained, increasing the diversity of the population. This implementation does not ensure just valid chromosomes because in some cases, non-valid chromosomes (non radial configurations) are obtained. However, in combination with the mutation, this disadvantage is transformed into an important advantage because the diversity of the population is substantially increased and new zones from the research space are explored. Not ultimately, the implemented inversion operator, applied a random number of times to the chromosomes, expands the search space enough in order to find a good solution in a reduced number of generations.

\section{Conclusions}

Reconfiguration represents one of the most important measures which can improve the performance in the operation of a distribution system. Optimization through the reconfiguration (or optimal reconfiguration) of a power distribution system is not a new problem but still represents a difficult one and nowadays has new valences. Besides active power losses, the average number of interruptions to the power supply represents an essential criterion which must be taken into consideration in the optimization problem. The criteria for optimization have been evaluated on active power distribution systems (containing distributed generators connected directly to the main distribution system and microgrids operated in grid-connected mode). The simulation studies on test systems have highlighted that the SAIFI index is smaller in the case of existing microgrids (because the consumers which are inside the microgrid are supplied even if a fault has occurred in the main distribution system) than in the case of distributed generators connected directly to the main distribution system.

The original formulation of the optimization problem, as a Pareto optimal one, with two objective functions (active power losses and system average interruption frequency index) ensures an objective and robust solution. Thus, the weak points of the classic methods proposed in literature can be eliminated; we are especially talking about: (i) errors caused by the conversion of different objective functions into the same measurement units; and (ii) the subjectivities caused by the introduction of weighting factors for different criteria.

Usually, the existing reconfiguration methods used nowadays either demand prohibitive execution times or result in non-optimal solutions (in the case of most common heuristics). The authors propose an original genetic algorithm (based on NSGA-II) to solve the problem (as a Pareto optimal one) in a non-prohibitive execution time. The comparative tests performed on some active test systems have demonstrated the accuracy and promptness of the proposed algorithm.

\section{Acknowledgments}

This work was supported by the project "Development and support of multidisciplinary postdoctoral programmes in major technical areas of national strategy of Research-Development-Innovation" 4D-POSTDOC, contract no. POSDRU/89/1.5/S/52603, a project co-funded by the European Social Fund through Sectoral Operational Programme Human Resources Development 2007-2013. We would 
like to thank the IREC — Catalonia Institute for Energy Research for hosting Bogdan Tomoiagă during his postdoctoral research stage. We would also like to thank Jennifer Fink for her English review.

\section{Appendix}

Table A1. Failure rates of system B (Figure 5b).

\begin{tabular}{|c|c|c|c|c|c|c|c|c|c|c|c|}
\hline Branch & $i$ & $j$ & $\lambda\left[\right.$ year $\left.^{-1}\right]$ & Branch & $i$ & $j$ & $\lambda\left[\right.$ year $\left.^{-1}\right]$ & Branch & $i$ & $j$ & $\lambda\left[\right.$ year $\left.^{-1}\right]$ \\
\hline 1 & B1 & 1 & 0.071 & 37 & 36 & 37 & 0.016 & 73 & B10 & 73 & 0.019 \\
\hline 2 & 1 & 2 & 0.096 & 38 & 37 & 38 & 0.024 & 74 & 73 & 74 & 0.082 \\
\hline 3 & 2 & 3 & 0.078 & 39 & 38 & 39 & 0.064 & 75 & 74 & 75 & 0.009 \\
\hline 4 & 3 & 4 & 0.035 & 40 & 39 & 40 & 0.065 & 76 & 75 & 76 & 0.091 \\
\hline 5 & 4 & 5 & 0.065 & 41 & 38 & 41 & 0.095 & 77 & B11 & 77 & 0.065 \\
\hline 6 & 5 & 6 & 0.082 & 42 & 41 & 42 & 0.046 & 78 & 77 & 78 & 0.017 \\
\hline 7 & 6 & 7 & 0.059 & 43 & B6 & 43 & 0.085 & 79 & 78 & 79 & 0.017 \\
\hline 8 & 7 & 8 & 0.012 & 44 & 43 & 44 & 0.075 & 80 & 79 & 80 & 0.091 \\
\hline 9 & 7 & 9 & 0.012 & 45 & 44 & 45 & 0.062 & 65 & B9 & 65 & 0.042 \\
\hline 10 & 7 & 10 & 0.011 & 46 & 45 & 46 & 0.036 & 66 & 65 & 66 & 0.054 \\
\hline 11 & B2 & 11 & 0.007 & 47 & B7 & 47 & 0.065 & 67 & 66 & 67 & 0.017 \\
\hline 12 & 11 & 12 & 0.039 & 48 & 47 & 48 & 0.093 & 68 & 67 & 68 & 0.016 \\
\hline 13 & 12 & 13 & 0.095 & 49 & 48 & 49 & 0.019 & 69 & 68 & 69 & 0.085 \\
\hline 14 & 12 & 14 & 0.036 & 50 & 49 & 50 & 0.065 & 70 & 69 & 70 & 0.066 \\
\hline 15 & B3 & 15 & 0.048 & 51 & 50 & 51 & 0.017 & 71 & 70 & 71 & 0.052 \\
\hline 16 & 15 & 16 & 0.076 & 52 & 51 & 52 & 0.017 & 72 & 71 & 72 & 0.052 \\
\hline 17 & 16 & 17 & 0.023 & 53 & 52 & 53 & 0.051 & 73 & B10 & 73 & 0.019 \\
\hline 18 & 17 & 18 & 0.094 & 54 & 53 & 54 & 0.026 & 74 & 73 & 74 & 0.082 \\
\hline 19 & 18 & 19 & 0.056 & 55 & 54 & 55 & 0.055 & 75 & 74 & 75 & 0.009 \\
\hline 20 & 19 & 20 & 0.067 & 56 & B8 & 56 & 0.026 & 76 & 75 & 76 & 0.091 \\
\hline 21 & 20 & 21 & 0.099 & 57 & 56 & 57 & 0.017 & 77 & B11 & 77 & 0.065 \\
\hline 22 & 21 & 22 & 0.045 & 58 & 57 & 58 & 0.032 & 78 & 77 & 78 & 0.017 \\
\hline 23 & 21 & 23 & 0.073 & 59 & 58 & 59 & 0.017 & 79 & 78 & 79 & 0.017 \\
\hline 24 & 23 & 24 & 0.046 & 60 & 59 & 60 & 0.068 & 80 & 79 & 80 & 0.091 \\
\hline 25 & B4 & 25 & 0.049 & 61 & 60 & 61 & 0.055 & 84 & 5 & 55 & 0.052 \\
\hline 26 & 25 & 26 & 0.062 & 62 & 61 & 62 & 0.061 & 85 & 7 & 60 & 0.051 \\
\hline 27 & 26 & 27 & 0.066 & 63 & 62 & 63 & 0.098 & 86 & 11 & 43 & 0.072 \\
\hline 28 & 27 & 28 & 0.059 & 64 & 63 & 64 & 0.017 & 87 & 12 & 72 & 0.061 \\
\hline 29 & 28 & 29 & 0.099 & 65 & B9 & 65 & 0.042 & 88 & 13 & 76 & 0.069 \\
\hline 30 & B5 & 30 & 0.034 & 66 & 65 & 66 & 0.054 & 89 & 14 & 18 & 0.049 \\
\hline 31 & 30 & 31 & 0.114 & 67 & 66 & 67 & 0.017 & 90 & 16 & 26 & 0.036 \\
\hline 32 & 31 & 32 & 0.074 & 68 & 67 & 68 & 0.016 & 91 & 20 & 83 & 0.045 \\
\hline 33 & 32 & 33 & 0.065 & 69 & 68 & 69 & 0.085 & 92 & 28 & 32 & 0.066 \\
\hline 34 & 33 & 34 & 0.059 & 70 & 69 & 70 & 0.066 & 93 & 29 & 39 & 0.085 \\
\hline 35 & 34 & 35 & 0.025 & 71 & 70 & 71 & 0.052 & 94 & 34 & 46 & 0.061 \\
\hline 36 & 35 & 36 & 0.026 & 72 & 71 & 72 & 0.052 & 95 & 40 & 42 & 0.042 \\
\hline
\end{tabular}




\section{References}

1. Merlin, A.; Back, H. Search for a Minimal-Loss Operating Spanning Tree Configuration in an Urban Power Distribution System. In Proceedings of the 1975 Fifth Power Systems Computer Conference (PSCC), Cambridge, UK, 1-5 September 1975; pp. 1-18.

2. Civanlar, S.; Grainger, J.J.; Yin, H.; Lee, S.S.H. Distribution feeder reconfiguration for loss reduction. IEEE Trans. Power Deliv. 1988, 3, 1217-1223.

3. Baran, M.E.; Wu, F.F. Network reconfiguration in distribution systems for loss reduction and load balancing. IEEE Trans. Power Deliv. 1989, 4, 1401-1407.

4. Shirmohammadi, D.; Hong, H.W. Reconfiguration of electric distribution networks for resistive line losses reduction. IEEE Trans. Power Deliv. 1989, 4, 1492-1498.

5. Chiang, H.D.; Jean-Jumeau, R. Optimal network reconfigurations in distribution systems: Part 2: Solution algorithms and numerical results. IEEE Trans. Power Deliv. 1990, 5, 1568-1574.

6. Nara, K.; Shiose, A.; Kitagawa, M.; Ishihara, T. Implementation of genetic algorithm for distribution systems loss minimum re-configuration. IEEE Trans. Power Systems 1992, 7 , 1044-1051.

7. Sárfi, R.J.; Salama, M.M.A.; Chinchani, A.Y. Distribution system reconfiguration: An algorithm based on network partitioning theory. IEEE Trans. Power Syst. 1996, 11, 504-510.

8. Sárfi, R.J.; Salama, M.M.A.; Chinchani, A.Y. System optimization of electrical distribution networks: A new nonlinear formulation. Int. J. Power Energy Syst. 1997, 17, 169-174.

9. Lin, W.M.; Chin, H.C. A new approach for distribution feeder reconfiguration for loss reduction and service restoration. IEEE Trans. Power Deliv. 1998, 13, 870-875.

10. Morton, A.; Mareels, M.Y. An efficient brute-force solution to the network reconfiguration problem. IEEE Trans. Power Deliv. 2000, 15, 996-1000.

11. Ramos, E.R.; Martinez-Ramos, J.L.; Exposito, A.G.; Salado, A.J.U. Optimal Reconfiguration of Distribution Networks for Power Loss Reduction. In Proceedings of the IEEE Porto Power Tech Conference, Porto, Portugal, 10-13 September 2001.

12. Radha, B.; Ah. King, R.T.F.; Rughooputh, H.C.S. Optimal Network Reconfiguration of Electrical Distribution Systems, In Proceedings of the International Conference on Industrial Technology (ICIT 2003), Maribor, Slovenia, 10-12 December 2003; pp. 66-71.

13. Oliveira, M.E.; Ochoa, L.F.; Padilha-Feltrin, A.; Mantovani, J.R.S. Network Reconfiguration and Loss Allocation for Distribution Systems with Distributed Generation. In Proceedings of the 2004 IEEE/PES Transmission \& Distribution Conference \& Exposition: Latin America, San Paulo, Brazil, 8-11 November 2004; pp. 206-211.

14. Queiroz, L.M.O.; Lyra, C. Adaptive hybrid genetic algorithm for technical loss reduction in distribution networks under variable demands. IEEE Trans. Power Syst. 2006, 24, 445-453.

15. Carpaneto, E.; Chicco, G. Distribution System Minimum Reconfiguration in the Hyper-Cube Ant Colony Optimization Framework. In Proceedings of the Sixth World Energy System Conference (WESC 2006), Torino, Italy, 10-12 July 2006; pp. 167-174.

16. Guimaraes, M.A.N.; Castro, C.; Romero, R. Reconfiguration of Distribution Systems by a Modified Genetic Algorithm. In Proceedings of the IEEE PowerTech'07, Lausanne, Switzerland, 1-5 July 2007. 
17. Yang, L.; Guo, Z. Reconfiguration of Electric Distribution Networks for Energy Losses Reduction. In Proceedings of the International Conference on Deregulation and Restructuring and Power Technologies (DRPT 2008), Nanjing, China, 6-9 April 2008; pp. 662-666.

18. Enacheanu, B.; Raison, B.; Caire, R.; Devaux, O.; Bienia, W.; Hadjsaid, N. Radial network reconfiguration using genetic algorithm based on the matroid theory. IEEE Trans. Power Syst. 2008, 23, 186-195.

19. Carreno, E.M.; Romero, R.; Padilha-Feltrin, A. An efficient codification to solve distribution network reconfiguration for loss reduction problem. IEEE Trans. Power Syst. 2008, 23, 1542-1551.

20. Chouhan, S.; Hui, W.; Lai, H.J.; Feliachi, A.; Choudhry, M.A. Intelligent Reconfiguration of Smart Distribution Network Using Multi-Agent Technology. In Proceedings of the 2009 IEEE Power and Energy Society General Meeting, Calgary, Canada, 26-30 July 2009; pp. 1-6.

21. Ababei, C.; Kavasseri, R. Efficient network reconfiguration using minimum cost maximum flow-based branch exchanges and random walks-based loss estimations. IEEE Trans. Power Syst. 2011, 26, 30-37.

22. Martinez-Rojas, M.; Sumper, A.; Gomis-Bellmunt, O.; Sudrià-Andreu, A. Reactive power dispatch in wind farms using particle swarm optimization technique and feasible solutions search. Appl. Energy 2011, 88, 4678-4686.

23. Roytelman, I.; Melnik, V.; Lee, S.S.H.; Lugtu, R.L. Multi-objective Feeder Reconfiguration by Distribution Management System. IEEE Trans. Power Syst. 1996, 11, 661-667.

24. Huang, Y.-C. Enhanced Genetic Algorithm-based Fuzzy Multi-objective Approach to Distribution Network Reconfiguration. IEE Proc. Gener. Transm. Distrib. 2002, 149, 615-620.

25. Asakura, T.; Genji, T.; Yura, T.; Hayashi, N.; Fukuyama, Y. Long-term distribution network expansion planning by network reconfiguration and generation of construction plans. IEEE Trans. Power Syst. 2003, 18, 1196-1204.

26. Tsai, M.-S.; Hsu, F.-Y. Application of grey correlation analysis in evolutionary programming for distribution system feeder reconfiguration. IEEE Trans. Power Syst. 2010, 25, 1126-1133.

27. Wu, Y.-K.; Lee, C.-Y.; Liu, L.-C.; Tsai, S.-H. Study of reconfiguration for the distribution system with distributed generators. IEEE Trans. Power Deliv. 2010, 25, 1678-1685.

28. Singh, D.; Misra, R.K. Multi-objective feeder reconfiguration in different tariff structures. IET Gener. Transm. Distrib. 2010, 4, 974-988.

29. Santos, A.C.; Delbem, A.C.B.; London, J.B.A.; Bretas, N.G. Node-depth encoding and multiobjective evolutionary algorithm applied to large-scale distribution system reconfiguration. IEEE Trans. Power Syst. 2010, 25, 1254-1265.

30. Guimaraes, M.A.N.; Castro, C.A.; Romero, R. Distribution systems operation optimisation through reconfiguration and capacitor allocation by a dedicated genetic algorithm. IET Gener. Transm. Distrib. 2010, 4, 1213-1222.

31. Mendoza, J.E.; Lopez, M.E.; Coello, C.A.; Lopez, E.A. Microgenetic multiobjective reconfiguration algorithm considering power losses and reliability indices for medium voltage distribution network. IET Gener. Transm. Distrib. 2009, 3, 825-840.

32. Bernardon, D.P.; Garcia, V.J.; Ferreira, A.S.Q.; Canha, L.N. Multicriteria distribution network reconfiguration considering subtransmission analysis. IEEE Trans. Power Deliv. 2010, 25, 2684-2691. 
33. Amanulla, B.; Chakrabarti, S.; Singh, S.N. Reconfiguration of power distribution systems considering reliability and power loss. IEEE Trans. Power Deliv. 2012, 27, 918-926.

34. Chiang, H.D.; Baran, M. On the existence and uniqueness of load flow solution for radial distribution power network. IEEE Trans. Circuits Syst. 1990, 37, 410-416.

35. IEEE Guide for Electric Power Distribution Reliability Indices. IEEE Standard 1366-2003, December 2003.

36. Heggie, G.; Yip, H.T. A Multi-Function Relay for Loss of Mains Protection. In Proceedings of the 1998 IEE Colloquium on System Implications of Embedded Generation and Its Protection and Control, London, UK, 25 February 1998; pp. 5/1-5/4.

37. Colet-Subirachs, A.; Ruiz-Alvarez, A.; Gomis-Bellmunt, O.; Alvarez-Cuevas-Figuerola, F.; Sudria-Andreu, A. Centralized and distributed active and reactive power control of a utility connected microgrid using IEC61850. IEEE Syst. J. 2012, 6, 58-67.

38. Tomoiagă, B.; Chindriş, M.; Sumper, A.; Sudrià-Andreu, A.; Marzband, M. Fuzzy Numbers Based Algorithm for Interruptions Frequency Estimation on Distribution Smart Grids. In Proceedings of the 2012 International Conference and Exposition on Electrical and Power Engineering (EPE 2012), Iasi, Romania, 25-27 October 2012; pp. 237-241.

39. Fonseca, C.; Fleming, P.J. Genetic Algorithms for Multiobjective Optimization: Formulation, Discussion, Generalization. In Proceedings of the 5th International Conference on Genetic Algorithms, San Mateo, CA, USA, 17-21 July 1993; pp. 416-423.

40. Horn, J.; Nafpliotis, N.; Goldberg, D.E. A Niched Pareto Genetic Algorithm for Multiobjective Optimization. In Proceedings of the 1st IEEE Conference on Evolutionary Computation, IEEE World Congress on Computational Intelligence, Piscataway, NJ, USA, 27-29 June 1994; Volume 1, pp. 82-87.

41. Zitzler, E.; Thiele, L.; Bader, J. On set-based multiobjective optimization. IEEE Trans. Evol. Comput. 2010, 14, 58-79.

42. Deb, K.; Pratap, A.; Agarwal, S.; Meyarivan, T. A fast and elitist multiobjective genetic algorithm: NSGA-II. IEEE Trans. Evol. Comput. 2002, 6, 182-197.

43. Goldberg, D.E.; Richardson, J. Genetic Algorithms with Sharing for Multimodal Function Optimization. In Proceedings of the 2nd International Conference on Genetic Algorithms and Their Applications, Hillsdale, NJ, USA, 28-31 July 1987; pp. 41-49.

(C) 2013 by the authors; licensee MDPI, Basel, Switzerland. This article is an open access article distributed under the terms and conditions of the Creative Commons Attribution license (http://creativecommons.org/licenses/by/3.0/). 